\title{
Application of Digital Image Processing in Drug Industry
}

\author{
Huvaida Manzoor
}

\begin{abstract}
Digital Image Processing is a very vast field It has many applications in every field and one such application is in drug industry. Drug Industry is leading industry. Manufacturing process in drug Industry is increasing day by day as we are dependent on these medicines. But these medicines might be defected, tablets may be broken, there may be missing tablet in a strip and consumption of such drugs might be dangerous. This paper shows different methods in digital image processing technique to find the defects in tablets. Four methods are used in order to detect the defect in tablet strip.
\end{abstract}

Keywords: Digital Image Processing, Morphology opening, Template Matching, Mathematical Manipulation, Euler's number

\section{Introduction}

Digital image processing techniques and algorithms are applied on images in order to remove error. In this paper, different digital image processing techniques are used to detect the defect in tablet strip $[1][2][4][5][6][7]$. Such defected tablets are harmful to consume and may have many side effects. The inspection process is effective to detect the defects in tablets. Four techniques are used that is morphology operation(MO), Template Matching(TM), Mathematical Manipulation(MM) and Euler's Method(EM) to defect the different defects in tablet strip. All the techniques are implemented in MATLAB 10. Figure 1 shows such defected tablet strips.

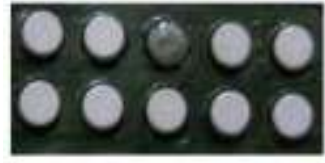

(a)

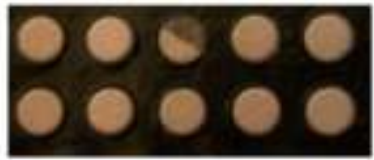

(b)

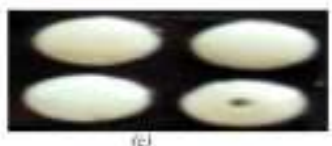

Figure 1 Different Tablet strips with defect (a) Missing Tablet (b) Broken Tablet (c) Hole in Tablet.

\section{Methodology}

a) Morphology Operation technique(MO)

b) Template Matching(TM)

c) Mathematical manipulation(MM)

d) Euler's Method(EM)

\section{Morphology Operation}

Morphological operations are affecting the form, structure or shape of an object. Applied on binary images (black \& white images - Images with only 2 colors: black and white). They are used in pre or post processing (filtering, thinning, and pruning) or for getting a representation or description of the shape of objects/regions (boundaries, skeletons convex hulls). Morphological operations apply a structuring element to an input image, creating an output image of the same size. In a morphological operation, the value of each pixel in the output image is based on a comparison of the corresponding pixel in the input image with its neighbors. By choosing the size and shape of the neighborhood, you can construct a morphological operation that is sensitive to specific shapes in the input image [1][3][8][11].

\section{Morphology is of following types:}

- Dilation: Dilation allows objects to expand, thus potentially filling in small holes and connecting disjoint objects.

- Erosion: Erosion shrinks objects by etching away (eroding) their boundaries.

- Opening: Morphological opening is used to remove small objects from an image while preserving the shape and size of larger objects in the image. Morphology opening is simply erosion followed by dilation. The opening has the effect of removing objects that cannot completely contain the structuring element.

- Closing: Closing is opening performed in reverse. It is defined simply as dilation followed by erosion. Erosion using the same structuring element for both operations. 


\section{Template Matching (TM)}

Technique used in classifying objects. Template matching techniques compare portions of images against one another[1][9][10][13]. Sample image may be used to recognize similar objects in source image. If standard deviation of the template image compared to the source image is small enough, template matching may be used. Templates are most often used to identify printed characters, numbers, and other small, simple objects. For example original image is of tablet packet containing ten tablets. Like morphology operation image is first converted into gray and then to binary. The binary image is then filtered in order to remove noise and edges are detected using canny edge detection. A template is selected and is compared with rest of the tablets In this way defected tablet can be found. The Template Matching block finds the best match of a template within an input image. The block computes match metric values by shifting a template over a region of interest or the entire image, and then finds the best match location.

\section{Mathematical Manipulation (MM)}

MM is used to find the defect in tablets.MM calculates the mathematical values. No. of tablets are calculated which helps to determine the correct figure in a tablet strip. Counting can also help to find the pharmaceutical company. They can simply discard the strip containing less or greater no. of tablets.

\section{Euler's Method(EM)}

Tablet which is having any hole is detected by using Euler's number of binary image. Euler's number is used to detect the holes in an object; we used this function in tablets having holes. Euler's number is the number of objects minus the number of holes [12].

$$
\mathrm{Eu}=\mathrm{N}-\mathrm{H}
$$

Where Eu,N and H are Euler's number, number of objects, number of holes respectively.

Also, $\mathrm{H}=\mathrm{N}-\mathrm{Eu}$

Therefore, from the above equation number of holes is equal to the number of objects minus the Euler's number. For finding Euler's number, image should be binary. Therefore, image is converted into binary. Euler's number tells whether tablet is having any hole or not. After finding Euler's number, position of tablet is detected. Also connected component of binary image is detected. Connected components in a binary image are a set of pixels that form a connected group. From this we also get image size, list of pixels belonging to each connected component. If the Euler's number is negative, it indicates that the number of holes is greater than the number of objects. Euler's number will detect whether the tablet is having any hole or not. After finding the presence of hole next step is to detect the position of hole. In order to detect the position of hole, we use simple arithmetic operation. Tablet with hole and without hole are subtracted.

\section{Proposed Work}

The proposed work is divided into four parts, that is

a. Morphology Operation technique(MO)

b. Template Matching(TM)

c. Mathematical manipulation(MM)

d. Euler's Method(EM)

All the above techniques are summarized in one block diagram as shown in figure 2

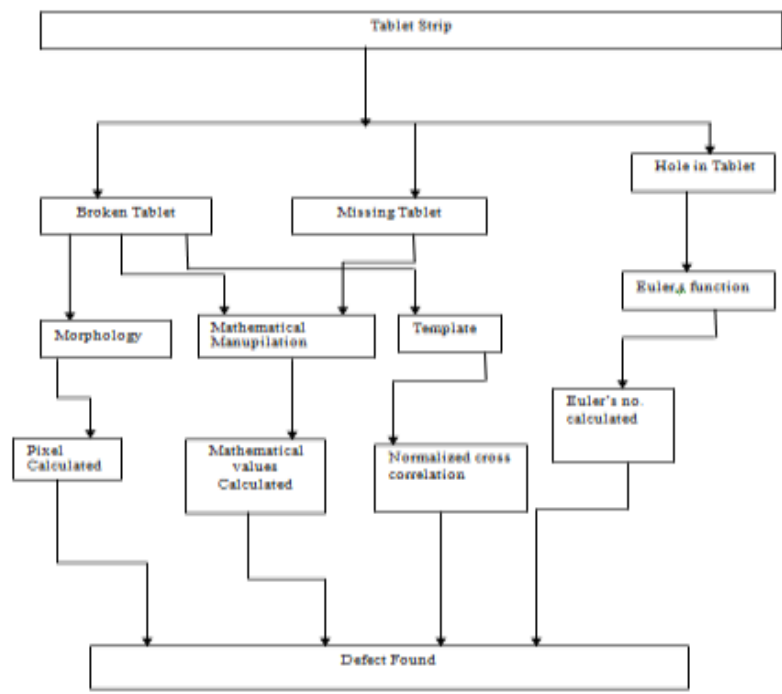

Figure 2 Block Diagram 
It is clearly shown in figure 2 that MO detects the broken tablet in tablet strip, TM, MM detects broken as well as missing tablet in tablet strip and EF detects hole in tablet strip.

\section{a) Steps for MO}

1. Capture an image of a tablet packet without any defect.

2. Convert this image into gray image.

3. Convert gray image into binary image

4. Apply morphology opening on binary image. Morphology opening will remove noise from the image.

5. Dilate the opened Image. Dilation will expand the image and will make the image big.

6. Calculate the no. of pixels of binary image.

7. Now capture the same tablet packet with broken/missing part.

8. Apply the same process that is applied on tablet packet without any defect.

9. When defected packet is found, beep will be generated.

10. Subtract the two images

11. Missing part of tablet and position of defect is obtained.

12. Filter it by using morphology opening technique which removes the noise.

\section{b) Steps for Template Matching}

1. Read an template image (defected or broken tablet).

2. Crop the image

3. Read the image of defected tablet packet.

4. Crop the image

5. Use normalized cross correlation to detect the position of defected tablet

6. Position of defected tablet found.

\section{c) Steps for $M M$}

1. Capture the image of tablet Strip

2. Convert the image into gray image

3. Convert gray image into binary image

4. Apply Morphology Opening on binary image. Morphology opening will remove noise from the image.

5. Boundaries of the output are detected after pre-processing.

6. After this process determine the roundness of the tablet, Find area and perimeter of each tablet

7. After calculating area and perimeter, find the metric. Metric closer to 1 indicates that tablet is not broken or is completely round.

\section{d) Steps for $E M$}

1. Input image is first pre-processed. Pre-processing involves conversion from RGB to gray image then to binary image. Binary image is then cleaned using morphology opening.

2. Pseudocolor is applied on opened image.

3. Connected objects is found.

4. Find the Euler's number.

5. If Euler's no. is equal to zero then the tablet is without hole else tablet is with hole and will ring beep.

6. Boundaries of the output are detected after pre processing.

7. After this process determine the roundness of tablet, find area and perimeter of each tablet.

8. After calculating area and perimeter, find the metric. Metric closer to 1 indicates that tablet is not broken or is completely round.

\section{Simulation Result of MO}

\section{Results And Discussion}

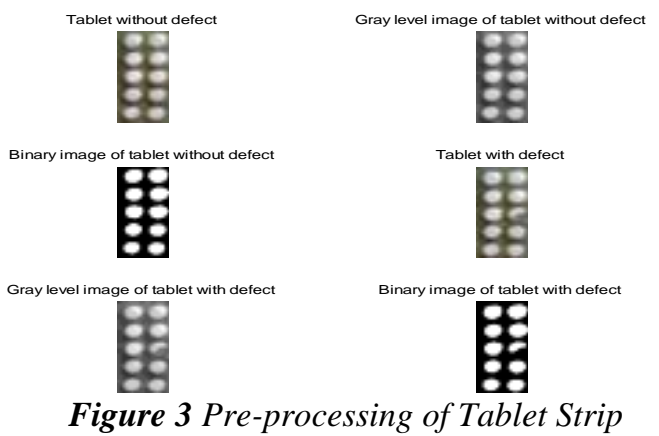



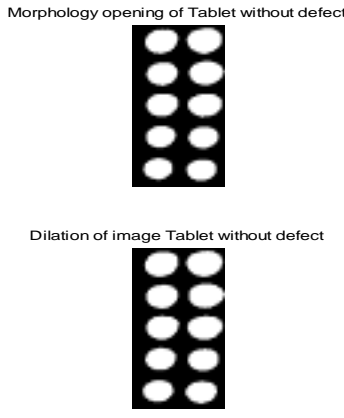

Figure 4 Morphology Opening process
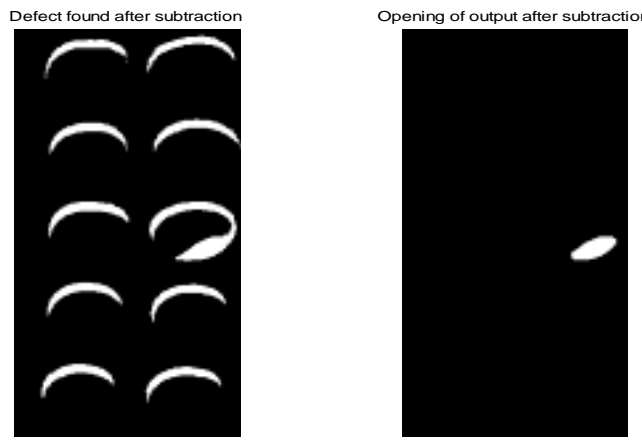

Figure 5 Defect detected

It is clearly shown in figure 3,4,5 how defected tablet is detected in tablet strip. At the end of program command window displays following information.

\begin{tabular}{|l|} 
Countwithout defect $=$ \\
342776 \\
Countwithdefect $=$ \\
348478 \\
TABLET RE.JECTED
\end{tabular}

Simulation Result of TM
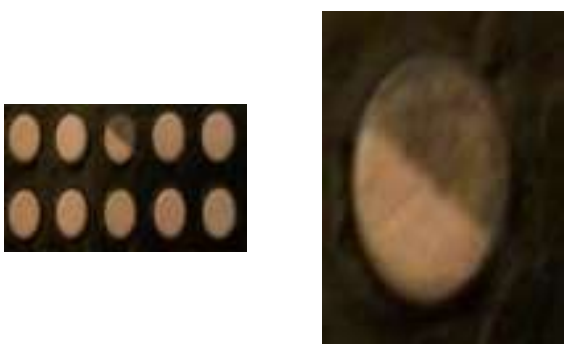

Figure 6 Tablet strip with broken part 

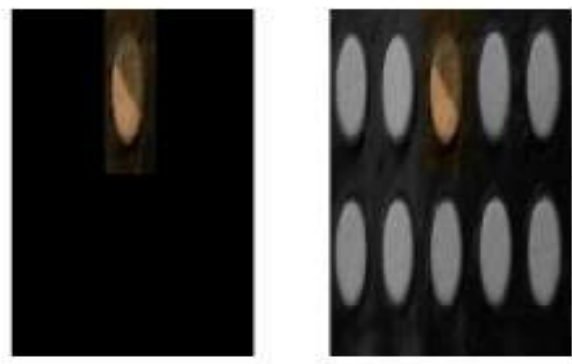

Figure 7 Broken tablet detected
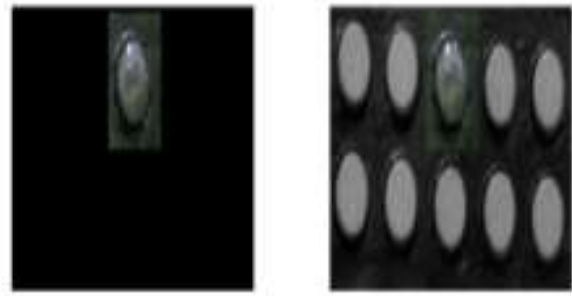

Figure 8 Missing Tablet detected

It is clear from figure 7 and 8 that template matching detects broken as well as missing tablet Simulation Result of MM

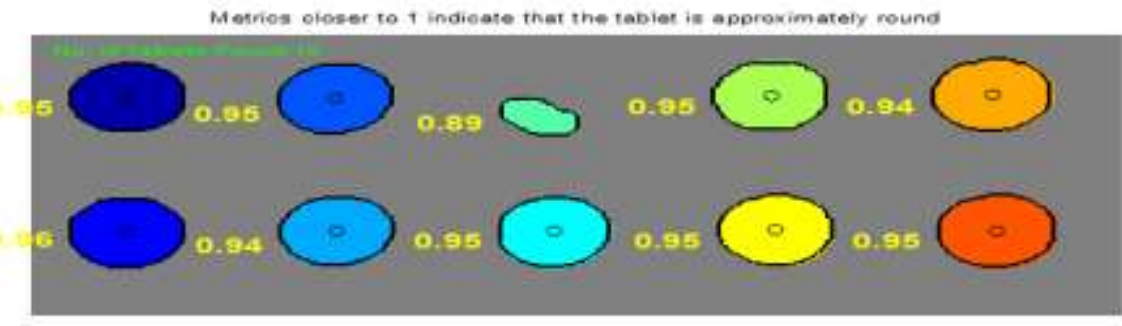

Figure 9 Shows defect detected by using MM

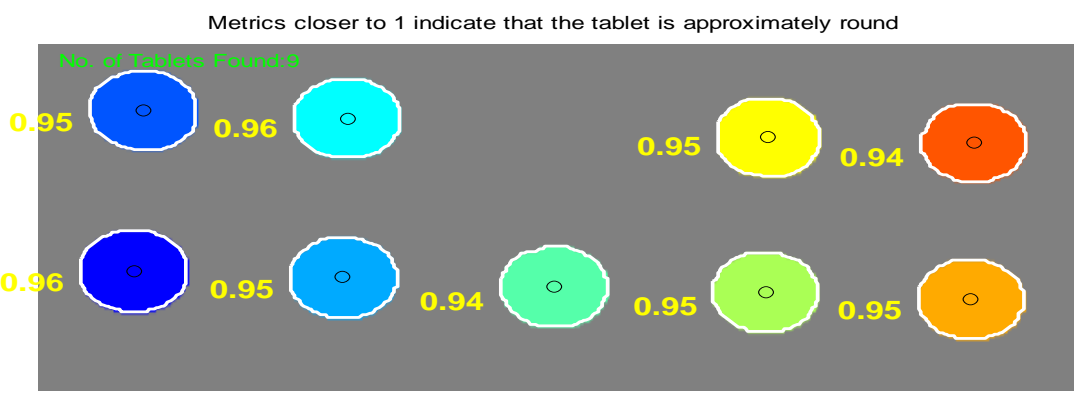

Figure 10 Missing Tablet detected in tablet strip

\section{Simulation Result of EF}
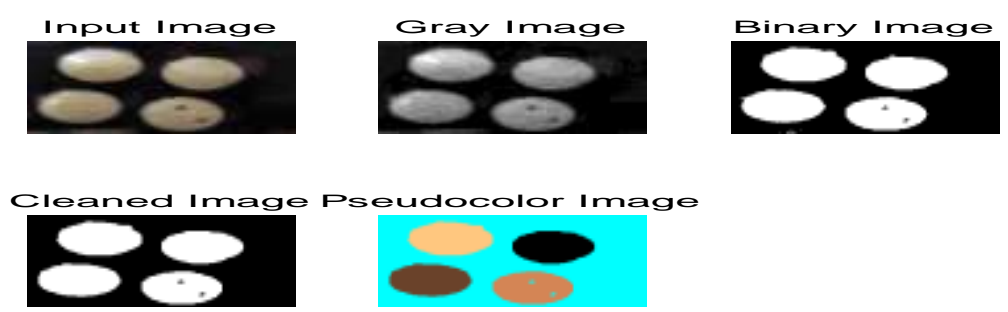

Figure 11 Holes in Tablet Strip 


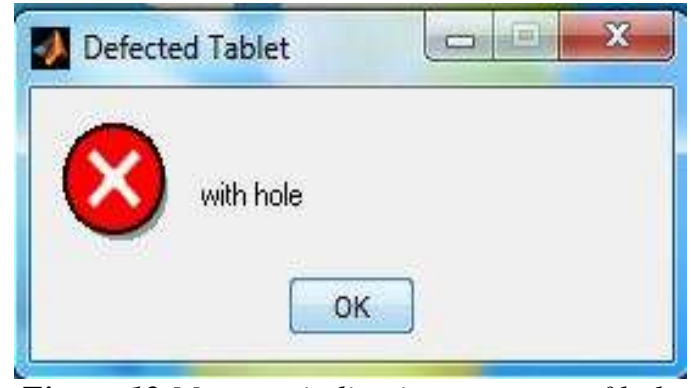

Figure 12 Message indicating presence of hole

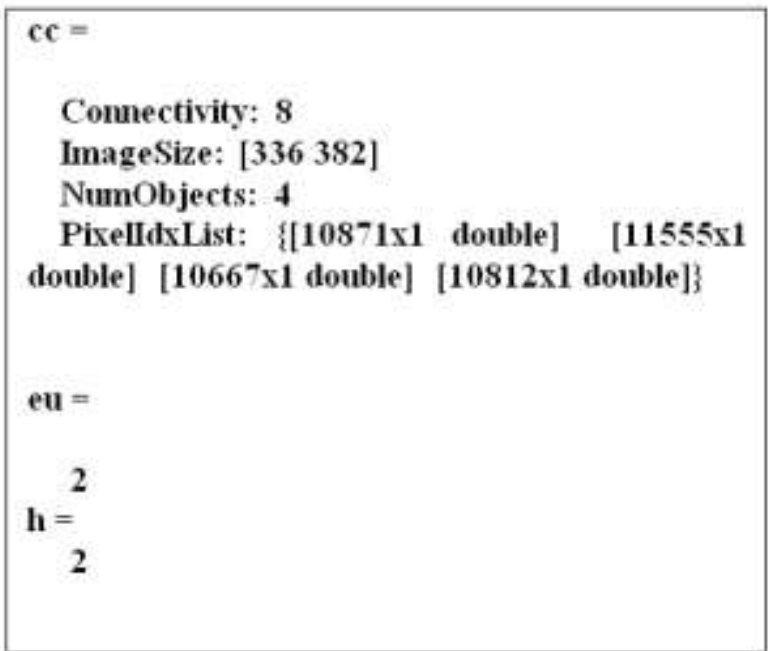

At the end of program command window is displayed with above information.

\section{Conclusion}

This paper presents the application in drug industry using digital image Processing technique. It is implemented on MATLAB 10. Four techniques are used that is MO, TM, MM and EF to defect the different defects in tablet strip. MO detects the broken tablet in tablet strip, TM, MM detects broken as well as missing tablet in tablet strip and EF detects hole in tablet strip.

\section{References}

[1]. Huvaida Manzoor, Yogeshwar SinghRandhawa-Comparative studies of algorithms using Digital Image Processing in Drug Industry -published at: "International Journal of Scientific and Research Publications (IJSRP), Volume 4,Issue 4,April 2014 Edition".

[2]. Manzoor,Huvaida, and Yogeshwer Singh Randhawa. "Edge Detection in Digital Image Using Statistical Method."

[3]. Ş̧efan Oprea, Ioan Liţă, Mariana Jurianu, Daniel Alexandru Vişan, Ion Bogdan Cioc, "Digital Image Processing Applied in Drugs Industry for Detection of Broken Aspirin Tablets" Electronics,Communications and Computers Department, University of Pitesti Str.Targul din Vale Nr. 1 Pitesti, Romania,2008

[4]. $\quad$ El-Khamy,S.E., Lotfy,M., El-Yamany,N.A.,“A Modified Fuzzy Sobel Edge Detector”,17. National Radio Science Conference, Feb.22-24 2000, Minufiya University, Egypt

[5]. Hardeep Kaur and Er.Nidhi Garg, "Inspection of Defective Pharmaceutical Capsules using Harris Algorithm", International Journal of Advances in Electronics Engineering,2009

[6]. Peng Zhao and Shutao Li "Tablets Vision Inspection Approach Using Fourier Descriptors and Support Vector Machines" The 9th International Conference for Young Computer Scientists,2008.

[7]. Ramya.S,,Suchitra.J,Nadesh R.K” Detection of Broken Pharmaceutical Drugs using Enhanced Feature Extraction Technique”

[8]. Baig, Hasan,Jeong-A. Lee, and Jieun Lee. "Performance evaluation of CPU-GPU and CPU-only algorithms for detecting defective tablets through morphological imaging techniques." Information Systems and Technologies (CISTI),2012 7th Iberian Conference on.IEEE, 2012.

[9]. Kitti, T., T. Jaruwan, and T. Chaiyapon. "An object recognition and identification system using the harris corner detection method." International Journal of Machine Learning and Computing 2.4 (2012): 462-465.

[10]. Zakaria, Mohd Firdaus, Hoo Seng Choon, and Shahrel Azmin Suand. "Object Shape Recognition in Image for Machine Vision Application." International Journal of Computer Theory \& Engineering 4.1 (2012).

[11]. Baig, Hasan, et al. "Implementation of SCADA system for unsought tablets detection through morphological image processing." Multitopic Conference, 2008. INMIC 2008. IEEE International. IEEE, 2008.

[12]. He, Li-Feng, Yu-Yan Chao, and Kenji Suzuki. "An algorithm for connected-component labeling, hole labeling and Euler number computing." Journal of Computer Science and Technology 28.3 (2013): 468-478.

[13]. Khalil, M. I. "Car plate recognition using the template matching method."International Journal of Computer Theory and Engineering 2.5 (2010): 1793-8201 